\title{
Rice false smut detection based on faster R-CNN
}

\author{
Prabira Kumar Sethy ${ }^{1}$, Nalini Kanta Barpanda ${ }^{2}$, Amiya Kumar Rath ${ }^{3}$, Santi Kumari Behera ${ }^{4}$ \\ ${ }^{1,2}$ Department of Electronics, Sambalpur University, India \\ ${ }^{3.4}$ Department of Computer Science and Engineering, Veer Surendra Sai University of Technology, India
}

\begin{tabular}{l}
\hline \hline Article Info \\
\hline Article history: \\
Received Jan 10, 2020 \\
Revised Mar 13, 2020 \\
Accepted Apr 6, 2020 \\
\hline Keywords: \\
Faster R-CNN \\
Object detection \\
Panicle DISEASES \\
Rice Crop \\
Rice false smut
\end{tabular}

Corresponding Author:

Prabira Kumar Sethy,

Department of Electronics,

Sambalpur University,

Jyoti Vihar, Burla, Odisha 768019, India.

Email: prabirsethy.05@gmail.com

\begin{abstract}
Rice false smut is one of the most dangerous diseases in rice at the ripening phase caused by Ustilaginoidea Virens. It is one of the most important grain diseases in rice production worldwide. Its epidemics not only lead to yield loss but also reduce grain quality because of multiple mycotoxins generated by the causative pathogen. The pathogen infects developing spikelets and specifically converts individual grain into rice false smut ball. Rice false smut balls seem to be randomly formed in some grains on a panicle of a plant in the paddy field. In this study, we suggest a novel approach for the detection of rice false smut based on faster R-CNN. The process of faster RCNN comprises regional proposal generation and object detection. The both tasks are done in same convolutional network. Because of such design it is faster for object detection. The faster R-CNN is able to detect the RFS using rectangular labelling from on-field images. The proposed approach is the initial steps to make a prototype for the automatic detection of RFS.
\end{abstract}

Copyright $@ 2020$ Institute of Advanced Engineering and Science. All rights reserved.

\section{INTRODUCTION}

Rice (Oryza sativa L.) is the primary food crop and consume as a staple food in Asia, Africa, South America, and to some extent in the United States [1]. More than $90 \%$ of the world's rice is developed and expanded in Asia, where 60\% of the total populace lives [2]. The production of rice is reduced mostly due to pest attacks and diseases. Among many diseases, RFS is one of the most harmful diseases. The RFS is caused by Ustilaginoidea virens and also known as pseudo-smut, or green smut. Earlier RFS is treated as a minor disease, but now it has been seen in many regions of the world [3-4]. It is a significant decimating fungal infection causing yield losses from 1.01 to $10.91 \%$ [5]. The RFS disease incidence has been recorded in India with the highest infection rate, i.e. $85 \%$ in Tamil Nadu [6-7].

In recent years, with the application of computer vision and machine learning [8-11], there has been an incredible advancement in disease diagnosis of crops such as detection, identification and quantification of various diseases. In most of the cases, SVM [12-13], K-Nearest neighbours (KNN) [14-15] and Discriminant analysis [16] are used for disease identification purposes. Many researchers reported automated rice disease diagnosis methods based on digital image processing technique [17], SVM [18], pattern recognition [19] and computer vision [20]. The research is not only for rice disease classification but also for other crops such as wheat [21], maize [22], cotton [23] and tomato [24] etc. Although, machine learning techniques have made great accomplishment in image identification, it has some limitations: restricted data handing capability, the requirement of segmentation \& feature extraction [25]. The diseased region segmentation is not always an easy task for all agricultural images [26]. Therefore, traditional machine learning techniques face difficulty in the classification of agriculturally diseased images with adequate results. In past few years, the CNN is applied in various fields such as object detection [27-31], image classification [32-34] and video 
classification [35]. In the last couple of years, many pieces of research have been conducted for the diagnosis of plant diseases based on CNN [35-39]. The objective of this study is to detect RFS based on faster R-CNN using on-field images of rice so that precaution can be taken.

\section{RESEARCH METHOD}

The methodology for the detection of RFS is detailed in the following subsections.

\subsection{Collection of rice false smut images}

The rice false smut images are collected from rice field with 48 Megapixel smartphone camera in natural daylight. The images captured with special care to blur the background by adjusting its secondary camera meant for focusing. Here we have collected 50 numbers of images only and use rotation (90 degrees, 180 degrees and 270 degrees) to increase the number of datasets. All the images are resized to a standard dimension, i.e. $227 \times 227 \times 3$.

\subsection{Image labelling}

The images are labelled with the fitting of the false smut affected region within the rectangular box. This labelling is done using image labeller app of Matlab 2019a. The defined rectangular regions of interest (ROI) labels are exported to the workspace of the main train algorithm in tabular form. The table contains the path of the images and its ROI label. The ROI label contains the four vertices of the rectangle, which encloses the false smut region.

\subsection{Train faster R-CNN}

The combination of feature extraction network and subsequent two sub-networks are called a regional proposal network (RPN). The RPN is used to train for the generation of proposals. The actual class of each proposal is predicted by the second sub-network. Here, the feature extraction is done by resnet50. Adjust the negative overlap range and positive overlap range to train the detector to ensure training samples are tightly overlapped with ground truth. The model of faster R-CNN is shown in Figure 1. The training parameters are detailed in Table 1.

Table 1. Detail of training parameter

\begin{tabular}{cc}
\hline Minibatch size & 1 \\
Validation frequency & 30 \\
Maximum epoch & 5 \\
Initial learning rate & 0.001 \\
Learning method & Stochastic gradient descent (SGDM) \\
\hline
\end{tabular}

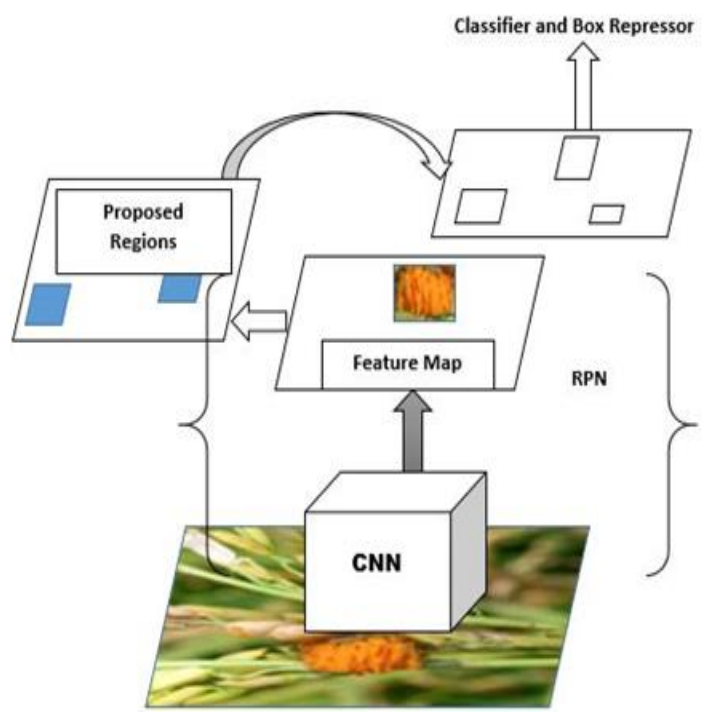

Figure 1. Faster R-CNN model for detection of RFS

\subsection{Detection of rice false smut}

After completion of detector training, the panicle images are fed for testing. The testing process is done using three different appearances of panicle image, i.e. immature healthy panicle, matured healthy panicle, and false smut affected panicle. The three different appearances of panicles are shown in Figure 2. 


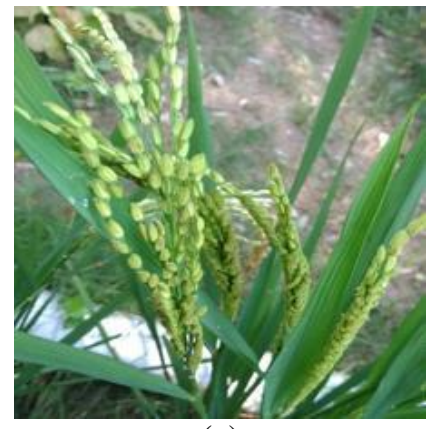

(a)

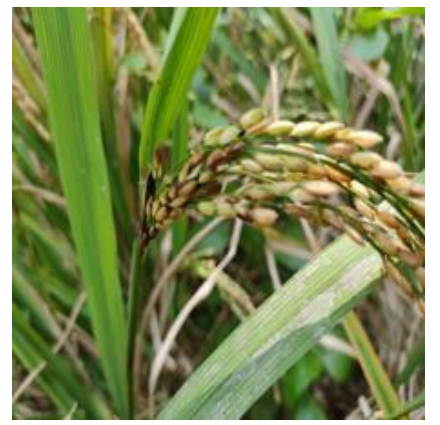

(b)

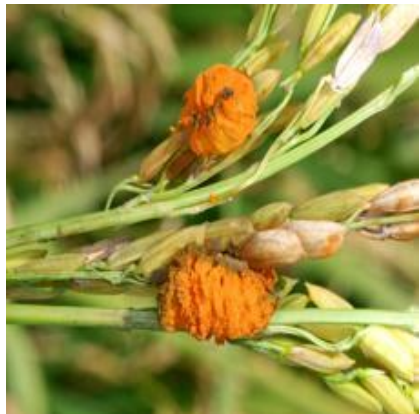

(c)

Figure 2. The different appearances of panicles (a) immature healthy panicle, (b) mature healthy panicle (c) false smut affected panicle

The detector of R-CNN returns the bounding box of the region affected by RFS with its score. If the summation of the score value is less or equal to zero, the panicle is healthy. If the summation of the score value is more than zero, the panicle is affected by false smut, and the affected region is enclosed by a rectangular bounding box.

\section{RESULTS AND DISCUSSION}

In this study, we detected the RFS using faster R-CNN. The experimental studies were implemented using the MATLAB 2019a deep learning toolbox. All applications were run on a laptop, i.e. Acer Predator Helios 300 Core i5 8th Gen - (8 GB/1 TB HDD/128 GB SSD/Windows 10 Home/4 GB Graphics) and equipped with NVIDIA GeForce GTX 1050Ti. The proposed method is examined using three appearances of panicles.

The first CNN provides the feature maps to the RPN. To make the region proposals, $3 \times 3$ filters slide over the feature map using a pre-trained network like resnet50. The output of resnet50 feeds to the fully connected layer to predict the boundary box and objectness score. The objectness ensures the presence of the object within the box and the regressor measures its score. Figure 3 shows the bounding box enclosing the false smut affected region with their scores. The RPN makes multiple guesses for a single location in the feature map, leading to multiple proposals for single objects, as in Figure 4(b). In contrast, the Faster R-CNN does not predict the random proposals, and makes a resemblance with the reference bounding box (called, Anchors) which makes way for successful detection. Again, for a single object, multiple numbers of Anchors are available with different shapes (one centred) which makes confusion to the detector to predict the proposals. The main reason for such a phenomenon may be, not to make bounding box proposals for each false smut affected region, like those shown in Figure 4 (a, c \& d). The different situation of anchors and proposals is illustrating in Figure 5.

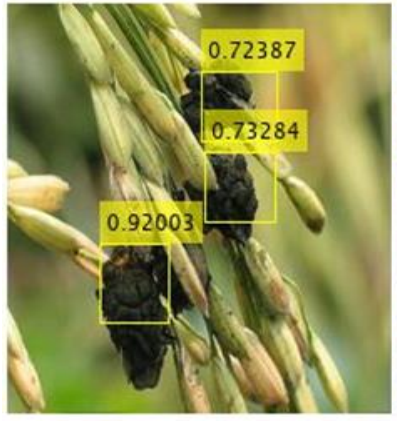

(a)

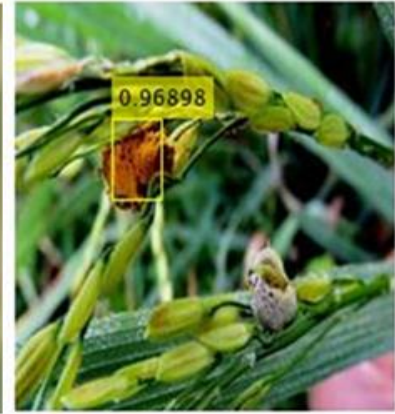

(b)

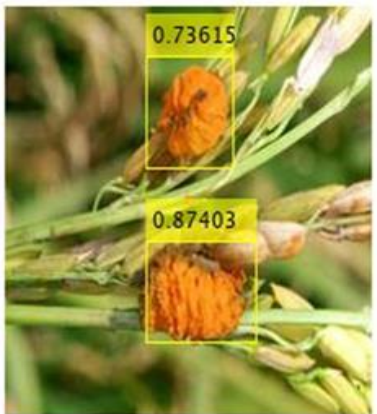

(c)

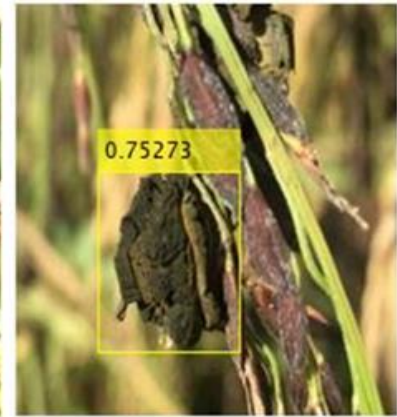

(d)

Figure 3. Successful detection of rice false smut with their score 


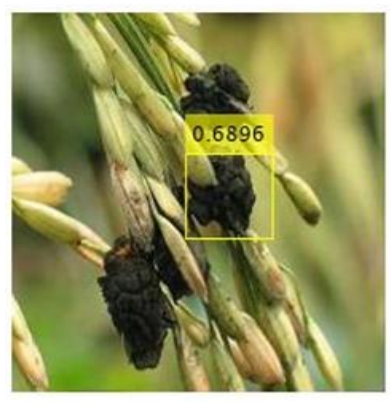

(a)

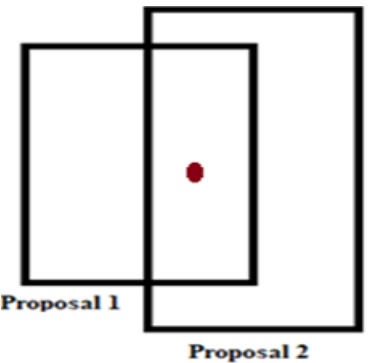

(a)

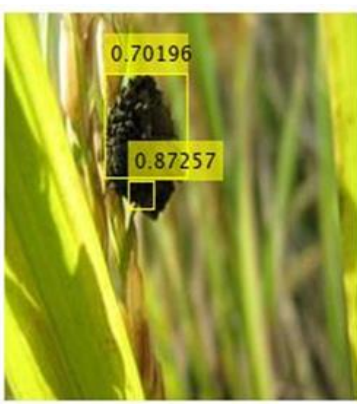

(b)

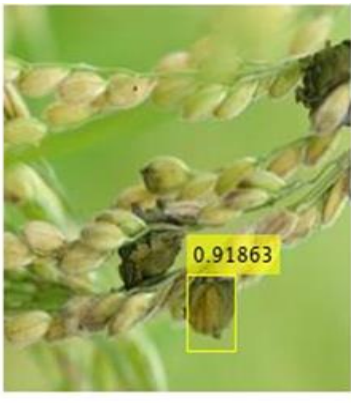

(c)

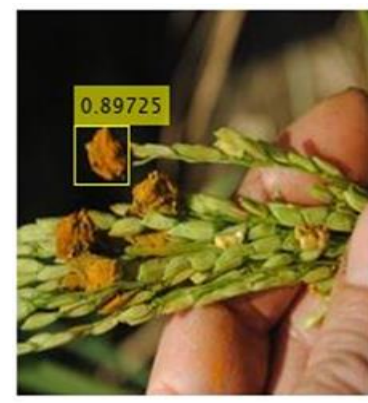

(d)

Figure 4. Ineffectual detection of rice false smut

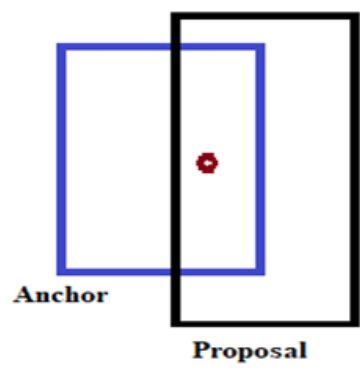

(b)

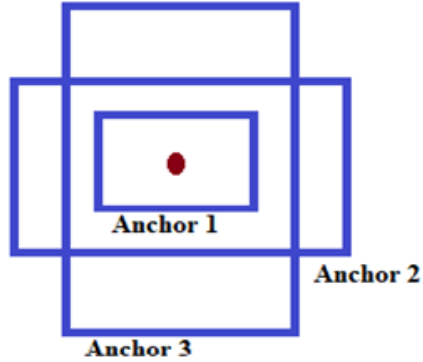

(c)

Figure 5. Different situation of anchors and proposals

The three main reasons for ineffectual detection are: (1) for one object location, multiple numbers of proposals are made, as shown in Figure 5 (a). (2) The shape of the proposal does not resemble with the Anchor, as shown in Figure 5(b). (3) The multiple numbers of Anchors are available for one object location, shown in Figure 5 (c). In the case of RFS detection, the ineffectual detection occurs due to touching and/ or overlapping of the false smut affected regions. The similar shape of bounding box labelling is made for different object locations leading to confusion to the detector.

\section{CONCLUSION}

This study proposes a method to detect rice false smut using on-field images. The faster R-CNN successfully detects the RFS, but in some cases, it leads to ineffectual detection. Therefore, the method of RFS detection needs to further improvement, even if false smut regions appear in complicated visual situations. Even if its limitation, this method is helpful to make a prototype for detection of rice false smut. In addition, this one is the first method for detection of rice false smut using computer intelligence instead of phytological diagnosis in state-of-art.

\section{REFERENCES}

[1] "Rice is Life" Food and Agricultural Organization of the United Nations. 2004. Retrieved, 2019.

[2] Guyer D, Tuttle A, Rouse S, Volrath S, Johnson M, Potter S, Görlach J, Goff S, Crossland L, Ward E. "Activation of latent transgenes in Arabidopsis using a hybrid transcription factor," Genetics, vol. 149, no. 2, pp. 633-9, 1998.

[3] Rush MC, Shahjahan AK, Jones JP, Groth DE. "Outbreak of false smut of rice in Louisiana," Plant disease, vol. 84, no. 1, pp. 100, 2000.

[4] Singh AK, Pophaly DJ. "An unusual rice false smut epidemic reported in Raigarh District", Chhattisgarh, India. Int. Rice Res. Notes, vol. 35, pp. 1-3, 2010.

[5] Atia MM. "Rice false smut (Ustilaginoideavirens) in Egypt," Journal of Plant Diseases and Protection, vol. 111, no. 1, pp. 71-82, 2004.

[6] Ladhalakshmi D, Laha GS, Singh R, Karthikeyan A, Mangrauthia SK, Sundaram RM, Thukkaiyannan P, Viraktamath BC. "Isolation and characterization of Ustilaginoideavirens and survey of false smut disease of rice in India," Phytoparasitica, vol. 40, no. 2, pp 171-176. 2012.

[7] Lu DH, Yang XQ, Mao JH, Ye HL, Wang P, Chen YP, He ZQ, Chen F. "Characterising the Pathogenicity Diversity of UstiiaginoideaVirens in Hybrid Rice in China,” Journal of Plant Pathology, vol. 91, no. 2, pp. 443-151, 2009. 
[8] Riri H. et al. "Extracted features based multi-class classification of orthodontic images," International Journal of Electrical and Computer Engineering, vol. 10, no. 4, pp. 3558-3567, 2020.

[9] Zulqarnain M. et al. "Text classification based on gated recurrent unit combines with support vector machine," International Journal of Electrical and Computer Engineering, vol. 10 no. 4, pp. 3734-3742, 2020. http://doi.org/10.11591/ijece.v10i4.pp3734-3742

[10] Noor D. et al. "Detecting abnormal movement of driver's head based on spatial-temporal features of video using deep neural network DNN," International Journal of Electrical and Computer Engineering, vol. 19, no. 1, pp. 344 352, 2020.

[11] Lee K.X. "Deep learning in non-coding variant," International Journal of Electrical and Computer Engineering. vol. 18, no. 3, pp. 1432-1438, 2020. http://doi.org/10.11591/ijeecs.v18.i3.

[12] Semary NA, Tharwat A, Elhariri E, Hassanien AE. "Fruit-based tomato grading system using features fusion and support vector machine,” In Intelligent Systems. Springer, Cham. pp. 401-410, 2014.

[13] Tian Y, Li T, Zhang L, Wang X. "Diagnosis method of cucumber disease with hyperspectral imaging in greenhouse," Transactions of the Chinese Society of Agricultural Engineering, vol. 26, no. 5, pp. 202-26, 2010.

[14] Prasad S, Peddoju SK, Ghosh D. "Multi-resolution mobile vision system for plant leaf disease diagnosis," Signal, Image and Video Processing, vol. 10, no. 2, pp. 379-388, 2016.

[15] Zhang SW, Shang YJ, Wang L. "Plant disease recognition based on plant leaf image," The journal of animal \& plant sciences, vol. 1, no. 25, pp, 42-45, 2015.

[16] Wang X, Zhang S, Wang Z, Zhang Q. "Recognition of cucumber diseases based on leaf image and environmental information," Transactions of the Chinese Society of Agricultural Engineering, vol. 30, no. 14 pp, 148-153, 2014

[17] Barbedo JG. "Digital image processing techniques for detecting, quantifying and classifying plant diseases," Springer Plus, vol. 2, no. 1, pp. 660, 2013.

[18] Jian Z, Wei Z. "Support vector machine for recognition of cucumber leaf diseases," In: IEEE 2nd international conference on advanced computer control, vol. 5, pp. 264-266, 2010.

[19] Phadikar S, Sil J. "Rice disease identification using pattern recognition techniques," In: IEEE 11th International Conference on Computer and Information Technology, pp. 420-423, Dec 2008.

[20] Asfarian A, Herdiyeni Y, Rauf A, Mutaqin KH. "A computer vision for rice disease identification to support Integrated Pest Management," Crop Protection, vol. 61, pp. 103-104, 2014

[21] Khairnar K, Dagade R. "Disease Detection and Diagnosis on Plant using Image Processing-A Review," International Journal of Computer Applications, vol. 108, no. 13, pp. 36-38, 2014.

[22] Zhang LN, Yang B. "Research on recognition of maize disease based on mobile internet and support vector machine technique," In Advanced Materials Research, Trans Tech Publications, vol. 905, pp. 659-662, 2014.

[23] Shicha Z, Hanping M, Bo H, Yancheng Z. "Morphological feature extraction for cotton disease recognition by machine vision," Microcomputer information, vol. 23, no. 4, pp. 290-292, 2007.

[24] Chai Y, Wang X. "Recognition of greenhouse tomato disease based on image processing technology," Pattern Recognition and Simulation, vol. 9, pp. 83-89, 2013.

[25] Chen L, Yuan Y. "Agricultural Disease Image Dataset for Disease Identification Based on Machine Learning," In International Conference on Big Scientific Data Management. Springer, Cham, pp. 263-274, 2018.

[26] Lu Y, Yi S, Zeng N, Liu Y, Zhang Y. "Identification of rice diseases using deep convolutional neural networks," Neurocomputing, vol. 267, pp. 378-84, 2017.

[27] Ren S, He K, Girshick R, Sun J. "Faster r-cnn: Towards real-time object detection with region proposal networks," In Advances in neural information processing systems, pp. 91-99, 2015.

[28] Girshick R, Donahue J, Darrell T, Malik J. "Rich feature hierarchies for accurate object detection and semantic segmentation," In Proceedings of the IEEE conference on computer vision and pattern recognition, pp. 580-587. 2014.

[29] "Girshick R. Fast r-cnn," Proceedings of the 2015 IEEE International Conference on Computer Vision. Santiago, Chile, pp. 1440-1448, 2015.

[30] Zitnick CL, "Dollár P. Edge boxes: Locating object proposals from edges," In European conference on computer visio, pp. 391-405, Sep 2014.

[31] Uijlings JR, Van De Sande KE, Gevers T, Smeulders AW. "Selective search for object recognition," International journal of computer vision, vol. 104, no. 2, pp. 154-171, 2013.

[32] Deng J, Dong W, Socher R, Li LJ, Li K, Fei-Fei L. "Imagenet: A large-scale hierarchical image database," In: 2009 IEEE conference on computer vision and pattern recognition, pp. 248-255, 2009.

[33] Krizhevsky A, Sutskever I, Hinton GE. "Imagenet classification with deep convolutional neural networks," In Advances in neural information processing systems, pp. 1097-1105, 2012.

[34] Simonyan K, Zisserman A. "'Very deep convolutional networks for large-scale image recognition," arXiv preprint arXiv, 1409. pp. 1556, 2014.

[35] Donahue J, Jia Y, Vinyals O, Hoffman J, Zhang N, Tzeng E, Darrell T. "Decaf: A deep convolutional activation feature for generic visual recognition," In International conference on machine learning, pp. 647-655. 2014.

[36] Karpathy A, Toderici G, Shetty S, Leung T, Sukthankar R, Fei-Fei L. "Large-scale video classification with convolutional neural networks," In Proceedings of the IEEE conference on Computer Vision and Pattern Recognition, pp. 1725-1732. 2014.

[37] Kawasaki Y, Uga H, Kagiwada S, Iyatomi H. "Basic study of automated diagnosis of viral plant diseases using convolutional neural networks," In International Symposium on Visual Computing, pp. 638-645. 2015.

[38] Zhang XJ, Lu YF, Zhang SH. "Multi-task learning for food identification and analysis with deep convolutional neural networks," Journal of Computer Science and Technology, vol. 31, no. 3, pp. 489-500, 2016.

[39] Zhao W S, Peng Y L, "Rice Diseases and Insect Pests Atlas," China Agriculture Press, Beijing, China, 2012. 


\section{BIOGRAPHIES OF AUTHORS}

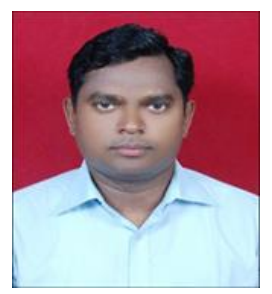

Prabira Kumar Sethy has received his Master of Technology degree in Communication Engineering from IIT (ISM) Dhanbad. He is working as Assistant Professor in Electronics, Sambalpur University, Odisha. His interest of research area is Agricultural Image Processing. He has published more than 40 number of research article in International Journal and Conferences.

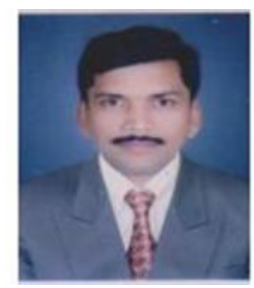

Nalini Kanta Barpanda has already received Ph.D. in Engineering from the Sambalpur University. He is working as Reader in Electronics, Sambalpur University, Odisha. He has published over 22 number of research articles in various areas of Performance Analysis of Communication Interconnection N/W, Wireless Sensor N/W, Image Processing, and Internet of Things.

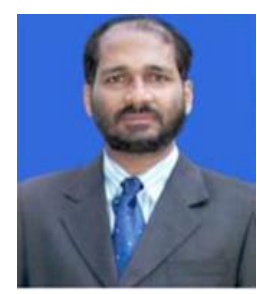

Amiya Kumar Rath has already received Ph.D. in Computer Science from the Utkal University, Odisha, India. He is working as Professor at Veer Surendra Sai University of Technology, Odisha. He has published over 70 number of research articles in various areas of Computer Science, concentrating on Artificial Intelligence, Image Processing, and Embedded System.

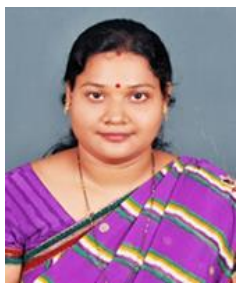

Santi Kumari Behera has received her Master of Technology degree in Computer Science \& Engineering from NIT Rourkela. She is working as Assistant Professor in Veer Surendra Sai University of Technology, Odisha, India. Her research of interest is Computer Graphics and Image Processing. 